\title{
A Fast Sensitivity Analysis Method For Voltage Control Applications With Distributed Generation
}

\author{
Khaled Alzaareer \\ École de technologie supérieure \\ Montreal, Quebec, Canada \\ khaled.alzaareer.1@ems.etsmtl.ca \\ Serge Lefebvre \\ Research Institute of Hydro-Quebec \\ Montreal, Quebec, Canada \\ sergelefebvrefcpq@ireq.ca
}

\author{
Maarouf Saad \\ École de technologie supérieure \\ Montreal, Quebec, Canada \\ Maarouf.saad@etsmtl.ca
}

\author{
Dalal Asber \\ Research Institute of Hydro-Quebec \\ Montreal, Quebec, Canada \\ Asber.Dalal@ireq.ca
}

\author{
Hasan Mehrjerdi \\ Qatar University \\ Doha, Qatar \\ Hasan.Mehrjerdi@qu.edu.qa
}

\begin{abstract}
Sensitivity Analysis has been widely used for voltage control of power grids. Typically, the sensitivity coefficients of network voltages are extracted from the inverse of Jacobian matrix. However, updating these coefficients based on the network operation conditions requires significant consuming time, which may add a new challenge for online voltage control of future power grids with distributed generation (DG), especially in the context of optimization techniques. In this paper, a fast sensitivity method is presented to find the sensitivity coefficients of network voltages via an analytical derivation. The approach is based on network admittance matrix and does not require extensive computing time, which is an advantage to be implemented in online applications. A $11 \mathrm{KV}$, 75-bus distribution network is used to verify the proposed method. A simple voltage control scenario is also presented to demonstrate the potential application of the proposed sensitivity method.
\end{abstract}

Index Terms-Sensitivity coefficients, distributed generation, distribution networks, voltage control, inverse of Jacobian matrix.

\section{INTRODUCTION}

Voltage control is one of the main problems in modern power networks and more specifically in the presence of DGs. Grid operators have to maintain the voltage magnitudes within acceptable limits. Sensitivity analysis plays a main role in optimal voltage control of power networks. The sensitivity coefficients are usually used to evaluate the voltages to the changes in the control variables.

One of the well-known techniques for sensitivity analysis of network voltages with respect to active or reactive power injections is the use of the coefficients gained by inverting Jacobian matrix [1],[2]. However, those coefficients are not constant. They usually vary with the change of system operating point and topology. Updating these coefficients based on network bases requires more consuming time, which may add a new challenge for real-time voltage control.

In [3], an approach based on the Gauss-Seidel method of load flow is developed. The approach depends on the impedance matrix of the network. However, this approach uses iterative process with a fixed number of iterations and, therefore, the accuracy of this method is low. In [4], the network impedance matrix was used with the constantcurrent model of loads to develop a sensitivity approach. However, this approach depends on the approximated representation of the network lines. In [5], an approach starring from branch currents are used for sensitivity analysis. However, this method requires a base load flow solution. In [6], A method based on the Tellegen theorem is developed to obtain sensitivity coefficients. However, this approach is based on the use of the so-called adjoint network.

As the penetration level of renewable distributed generation is increased in power networks, the need to develop optimal voltage control techniques in real time is raised. One of the requirements for optimal voltage control is to find a very fast solution.

With the motivation to develop a fast and accurate method for sensitivity analysis and suitable for any network (transmission or distribution, balanced or unbalanced, radial or mesh networks), this work proposes an approach for sensitivity analysis. The main contribution of this work is to provide an analytical derivation of network voltages with respect to the active and reactive power injections. The method is based on the network admittance matrix and can be considered as a good alternative method for traditional ones (i.e. using the inverse of Jacobian matrix). In this work, a simple voltage control scenario is also presented to demonstrates the potential application of the proposed approach.

The rest of the paper is as follows. Section II shows the proposed sensitivity analysis to find the sensitivity coefficients of network voltages with respect to power injection. Section III shows simulation results of the case study while Section V states the conclusions.

\section{Fourmulation OF SENSITIVITY COEFFICIENTS Via ANALYTICAL DERIVATION}

To find the sensitivity coefficients of network voltages to power injections, let us consider the simple network shown in Fig.1. P+jQ represents the load of bus 2 while V and I represent the load voltage and current, respectively. Suppose that the amount $\Delta \mathrm{P}$ or $\Delta \mathrm{Q}$ is incrementally injected into the bus 2 . This power injection will increase the voltage by $\Delta \mathrm{V}$. Thus, the new voltage of bus 2 can be written as $\mathrm{V}+\Delta \mathrm{V}$. Similarly, the node current will also be changed by an amount $\Delta \mathrm{I}$, making the new bus injected current $\mathrm{I}+\Delta \mathrm{I}$.

Let the load at bus 2 at the $1^{\text {st }}$ instant be $S_{1}$ and the load at the $2^{\text {nd }}$ instant be $S_{2}$ as follows:

$$
\begin{aligned}
& S_{1}=V I^{*} \\
& S_{2}=(V+\Delta V)\left(I^{*}+\Delta I^{*}\right)
\end{aligned}
$$

Then, the change in complex power $\Delta \mathrm{S}$ at bus 2 can now obtained as:

$$
\begin{aligned}
\Delta S & =S_{2}-S_{1} \\
& =(V+\Delta V)\left(I^{*}+\Delta I^{*}\right)-V I^{*} \\
& =V \Delta I^{*}+\Delta V \Delta I^{*}+I^{*} \Delta V
\end{aligned}
$$




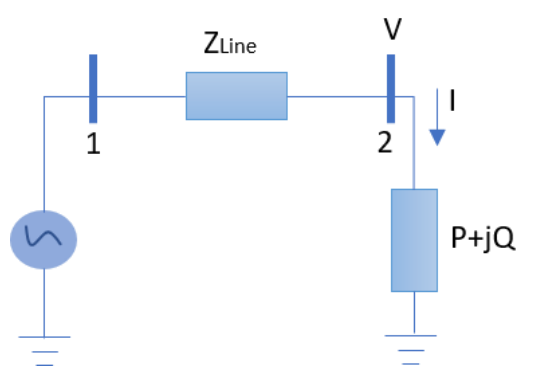

Fig.1. Simple system.

It is worth mentioning that the smaller the value of $\Delta \mathrm{P}$ or $\Delta \mathrm{Q}$ (implying $\Delta \mathrm{V} \& \Delta \mathrm{I}$ are also small), the closer the two operating points are the better the estimation of the sensitivity coefficients. The second term of (2) represents a very small value and, thus, can be neglected. Accordingly, (2) becomes:

$$
\Delta S=V \Delta I^{*}+I^{*} \Delta V
$$

dividing (3) by $\Delta P$ or $\Delta Q$ yields:

$$
\begin{aligned}
& \frac{\Delta S}{\Delta P}=V \frac{\Delta I^{*}}{\Delta P}+I^{*} \frac{\Delta V}{\Delta P} \\
& \frac{\Delta S}{\Delta Q}=V \frac{\Delta I^{*}}{\Delta Q}+I^{*} \frac{\Delta V}{\Delta Q}
\end{aligned}
$$

By evaluating the limit of the expression in (4) as $\Delta P($ or $\Delta Q) \rightarrow 0$, the terms $\frac{\Delta V}{\Delta P}, \frac{\Delta V}{\Delta Q}, \frac{\Delta I^{*}}{\Delta P}, \frac{\Delta I^{*}}{\Delta Q}, \frac{\Delta S}{\Delta P}$ and $\frac{\Delta S}{\Delta Q}$ become $\frac{\partial V}{\partial P}, \frac{\partial V}{\partial Q}, \frac{\partial I^{*}}{\partial P}, \frac{\partial I^{*}}{\partial Q}, \frac{\partial S}{\partial P}$ and $\frac{\partial S}{\partial Q}$ respectively. Thus, the expression in (4) can be expressed as:

$$
\begin{aligned}
& \frac{\partial S}{\partial P}=V \frac{\partial I^{*}}{\partial P}+I^{*} \frac{\partial V}{\partial P} \\
& \frac{\partial S}{\partial Q}=V \frac{\partial I^{*}}{\partial Q}+I^{*} \frac{\partial V}{\partial Q}
\end{aligned}
$$

The formulas in (5) can be extended for multi-bus networks. For any bus i of the PQ buses, the derivation in (5) becomes:

$$
\begin{aligned}
& \frac{\partial S_{i}}{\partial P_{x}}=V_{i} \frac{\partial I_{i}{ }^{*}}{\partial P_{x}}+I_{i}{ }^{*} \frac{\partial V_{i}}{\partial P_{x}} \\
& \frac{\partial S_{i}}{\partial Q_{x}}=V_{i} \frac{\partial I_{i}{ }^{*}}{\partial Q_{x}}+I_{i}{ }^{*} \frac{\partial V_{i}}{\partial Q_{x}}
\end{aligned}
$$

Where $\frac{\partial S_{i}}{\partial P_{x}}$ and $\frac{\partial S_{i}}{\partial Q_{x}}$ represent the partial derivatives of the complex power of bus $i$ with respect to active and reactive power injection into the bus $\mathrm{x}$, respectively. $\frac{\partial V_{i}}{\partial P_{x}}, \frac{\partial V_{i}}{\partial Q_{x}}$ and $\frac{\partial I_{i}{ }^{*}}{\partial P_{x}}, \frac{\partial I_{i}{ }^{*}}{\partial Q_{x}}$ represent the partial derivatives of node voltage and current (conjugate) of bus i respectively with respect to active and reactive power injection into the bus $\mathrm{x} . V_{i}$ and $I_{i}{ }^{*}$ represent the node voltage and current conjugate of bus $i$.

This work assumes that the power injection at a particular bus is independent of power injections of other buses. Therefore if $\mathrm{i}=\mathrm{x}$, the sensitivities $\frac{\partial S_{i}}{\partial P_{x}}=1$ and $\frac{\partial S_{i}}{\partial Q_{x}}=-\mathrm{j} 1$. Otherwise, both sensitivities equal to zero.

The sensitivities $\frac{\partial I_{i}{ }^{*}}{\partial P_{x}}$ and $\frac{\partial I_{i}{ }^{*}}{\partial Q_{x}}$ can be easily found if the current conjugate is written in terms of the network admittances and node voltages as given in (7). $\mathrm{Y}$ is the network bus admittance, I and $\mathrm{V}$ are bus current and voltage vectors, respectively.

$$
[I]=[Y][V]
$$

Considering that $\mathrm{N}$ is the number of network buses while $\mathrm{M}$ is the number of PQ buses, the injected current of bus i can be expressed as:

$$
I_{i}{ }^{*}=\sum_{\mathrm{j} \in N} Y_{i j}{ }^{*} V_{j}{ }^{*}, \quad i \in M
$$

Thus, the sensitivities $\frac{\partial I_{i}{ }^{*}}{\partial P_{x}}$ and $\frac{\partial I_{i}{ }^{*}}{\partial Q_{x}}$ can be written as:

$$
\begin{array}{ll}
\frac{\partial I_{i}^{*}}{\partial P_{x}}=\sum_{\mathrm{j} \in M} Y_{i j}{ }^{*} \frac{\partial V_{j}^{*}}{\partial P_{x}}, & i \in M \\
\frac{\partial I_{i}^{*}}{\partial Q_{x}}=\sum_{\mathrm{j} \in M} Y_{i j}{ }^{*} \frac{\partial V_{j}^{*}}{\partial Q_{x}}, & i \in M
\end{array}
$$

By substituting (10) into (6), (6) becomes:

$$
\begin{aligned}
& \frac{\partial S_{i}}{\partial P_{x}}=V_{i} \sum_{\mathrm{j} \in M} Y_{i j}{ }^{*} \frac{\partial V_{j}^{*}}{\partial P_{x}}+\frac{\partial V_{i}}{\partial P_{x}} \sum_{\mathrm{j} \in N} Y_{i j}{ }^{*} V_{j}{ }^{*} \\
& \frac{\partial S_{i}}{\partial Q_{x}}=V_{i} \sum_{\mathrm{j} \in M} Y_{i j}{ }^{*} \frac{\partial V_{j}^{*}}{\partial Q_{x}}+\frac{\partial V_{i}}{\partial Q_{x}} \sum_{\mathrm{j} \in N} Y_{i j}{ }^{*} V_{j}{ }^{*}
\end{aligned}
$$

Equation (10) can be performed for each bus i $\epsilon M$. To find the partial derivatives $\frac{\partial V_{i}}{\partial P_{x}}$ and $\frac{\partial V_{i}}{\partial Q_{x}}$, the later system of equations is solved.

From (10), it can be seen that the proposed method depends on parameters of Y bus (i.e. topology of the system). It can also be clear that the sensitivity approach is computed via the derivation of the nodal voltages with respect to the power injections.

Similar equations to (10) can be used to find the partial derivatives with respect to other power injections (i.e. for power injections at node $\mathrm{x} \in N$ ). It is worth mentioning that system of equations is the same regardless at which bus the power is injected. The only change is in the value of $\frac{\partial S_{i}}{\partial P_{x}}$ or $\frac{\partial S_{i}}{\partial Q_{x}}$.

Once the partial derivatives $\frac{\partial V_{i}}{\partial P_{x}}$ and $\frac{\partial V_{i}}{\partial Q_{x}}$ are obtained, the sensitivities of the voltage magnitude $\frac{d\left|V_{i}\right|}{d P_{x}}$ and $\frac{d\left|V_{i}\right|}{d Q_{x}}$ can be obtained as:

$$
\begin{array}{r}
\frac{d\left|V_{i}\right|}{d P_{x}}=\frac{1}{\left|V_{i}\right|} \\
+\operatorname{Re}\left(V_{i}\right) \frac{d\left(\operatorname{Re}\left(V_{i}\right)\right)}{d P_{x}} \\
\left.+\operatorname{Im}\left(V_{i}\right) \frac{d\left(\operatorname{Im}\left(V_{i}\right)\right)}{d P_{i}}\right) \\
\frac{d\left|V_{i}\right|}{d Q_{x}}=\frac{1}{\left|V_{i}\right|}\left(\operatorname{Re}\left(V_{i}\right) \frac{d\left(\operatorname{Re}\left(V_{i}\right)\right)}{d Q_{x}}\right. \\
\left.+\operatorname{Im}\left(V_{i}\right) \frac{d\left(\operatorname{Im}\left(V_{i}\right)\right)}{d Q_{x}}\right)
\end{array}
$$

The sensitivities $\frac{d\left(\operatorname{Re}\left(V_{i}\right)\right)}{d P_{x}}, \frac{d\left(\operatorname{Im}\left(V_{i}\right)\right)}{d P_{x}}$ and $\frac{d\left(\operatorname{Re}\left(V_{i}\right)\right)}{d Q_{x}}, \frac{d\left(\operatorname{Im}\left(V_{i}\right)\right)}{d Q_{x}}$ can be directly obtained from:

$$
\begin{aligned}
& \frac{d V_{i}}{d P_{x}}=\frac{d\left(\operatorname{Re}\left(V_{i}\right)\right)}{d P_{x}}+j \frac{d\left(\operatorname{Im}\left(V_{i}\right)\right)}{d P_{x}} \\
& \frac{d V_{i}}{d Q_{x}}=\frac{d\left(\operatorname{Re}\left(V_{i}\right)\right)}{d Q_{x}}+j \frac{d\left(\operatorname{Im}\left(V_{i}\right)\right)}{d Q_{x}}
\end{aligned}
$$

\section{SimULATION RESULTS}

To verify the applicability and robustness of the proposed approach for the computation of voltage sensitivities, 77-bus, $11 \mathrm{kV}$ distribution grid shown in Fig.2 is used as a test system. The network consists of 22 DG units and 53 load bus. In this paper, it is assumed that no output power by DG units. 
Since DG units can change the voltages by injecting power, the sensitivity values are obtained for all load buses with respect to power injection. This is to make a better verification of the proposed method. The system data can be found in [8]. MATLAB software is used to investigate the results.

\section{A. Calculation of the sensitivity coefficients of network voltages via the proposed analytical derivation}

The $d|V| / d P$ and $d|V| / d Q$ sensitivities of the test system are calculated at the base-load condition and demonstrated in Fig. 3 and Fig. 4, respectively. It is clear from the results that sensitivity values are non-negative. This means that injecting reactive power into the system will increase the voltages. We can also notice that the self-sensitivities in both figures are higher than the cross-sensitivities. In other words, injecting power at a particular node can increase the voltage at its own location more than the voltages of other nodes due to the effect of power flow across network impedances. It is also noticed that the voltage sensitivity of a particular bus to power injection at other buses in the same feeder is higher than the sensitivity due to power injection at the buses of other feeders.

According to the power flow calculation under the base load condition, it was found that bus 1175 has the lowest voltage magnitude (critical bus). The $d|V| / d P$ and $d|V| / d Q$ sensitivities of the critical bus, bus 1175, are shown in Fig.5. We can see that the voltage sensitivity of bus 1175 to active or reactive power injected at bus 1175 (i.e. $\mathrm{Q}_{1175}$ or $\mathrm{P}_{1175}$ ) is higher than the voltage sensitivities of bus 1175 to power injection at other nodes. Moreover, the sensitivity to active or reactive power injected at bus 1165 (i.e. $\mathrm{Q}_{1165}$ or $\mathrm{P}_{1165}$ ) is the second largest among all the sensitivities. This can be demonstrated from the analytical expressions illustrated in (10) and (11). It can also be seen that the sensitivities are related to the coupling admittances between network buses. In our example, bus 1165 is the nearest node to bus 1175 among all other nodes. Thus, the voltage sensitivity of bus 1175 to power injection at 1165 is higher than its sensitivities due to the power injection at other buses (i.e. the largest cross-sensitivity value is obtained with bus 1165).

Verification of the voltage sensitivities of bus 1175 to active and reactive power injection at its own bus (selfsensitivity) and bus 1165 (the largest cross-sensitivity) demonstrates the validity of the proposed sensitivities.

\section{B. Verification of the obtained sensitivity coefficients of network voltages}

In this subsection, the verification of voltage sensitivities $d|V| / d P$ and $d|V| / d Q$ of bus 1175 to power injection at node 1175 (self-sensitivity) and bus 1165 (largest crosssensitivity) are investigated in detail.

In order to verify the self-sensitivity $d|V| / d P$ of node $1175,1.0$ p.u of its own active power load with step 0.1 p.u are injected into the bus 1175 . The voltage magnitudes of node 1175 are then predicted by multiplying the selfsensitivity $d|V| / d P$ at the base condition with the amount of the active power injection. These voltages are then compared with the actual voltages obtained via the results of power flow with the active power injection. A similar verification is repeated for the largest cross-sensitivity $d|V| / d P$ of node 1175. 1.0 p.u of node 1165 active power load with step 0.1 p.u are injected into the bus 1165 . Fig.6 (a) and Fig.6 (b) show the verification of $d|V| / d P$ sensitivity of bus 1175 due to active power injected at bus 1175 and bus 1165 respectively.

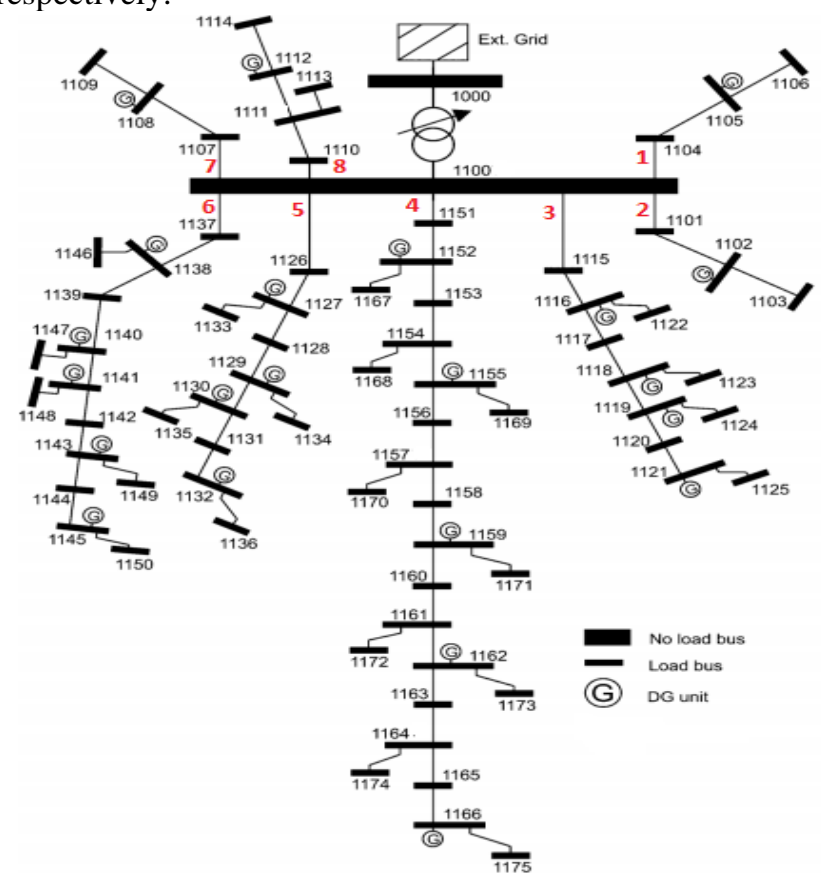

Fig.2: Topology of the test system [7]

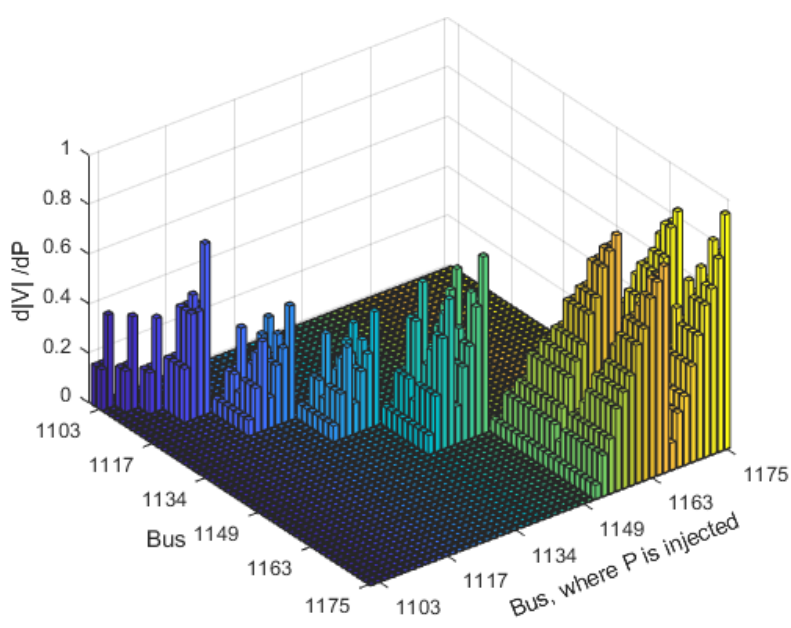

Fig.3. $d|V| / d P$ sensitivities at base load condition.

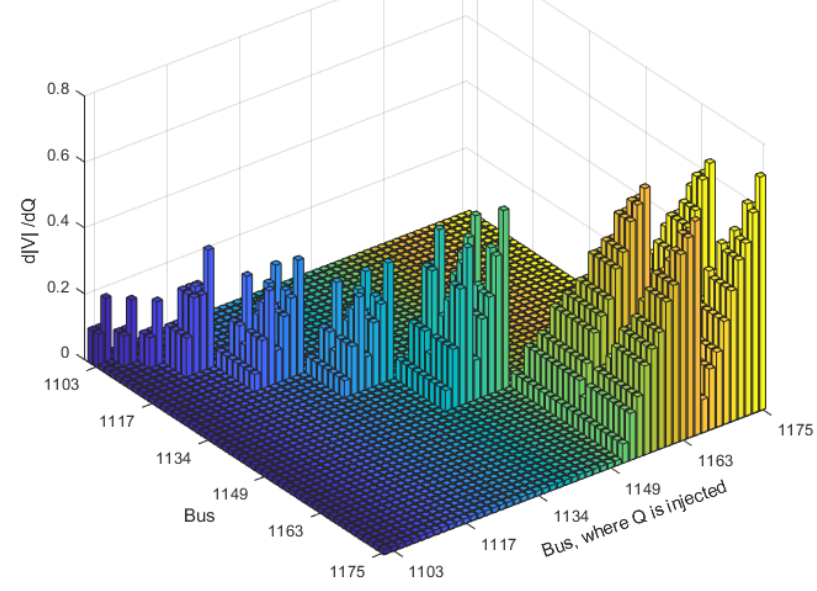

Fig.4. $d|V| / d Q$ sensitivities at base load condition. 


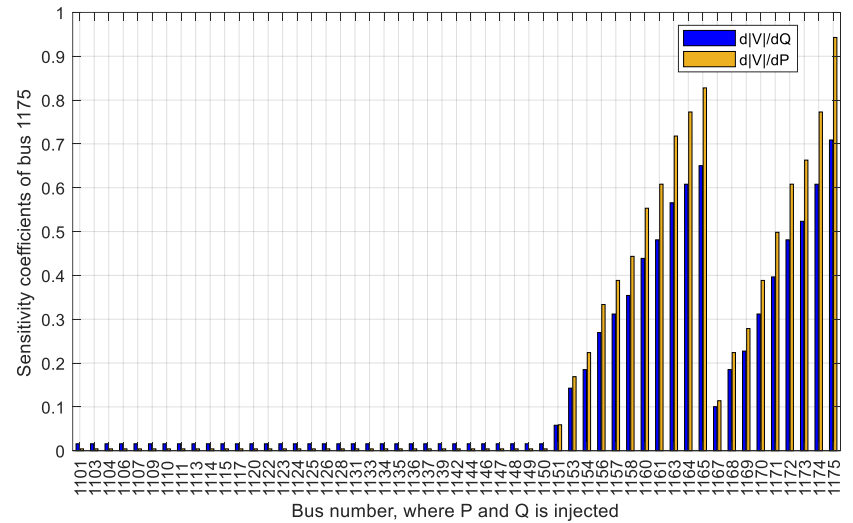

Fig.5. Sensitivity coefficients of bus 1175 with respect to active and reactive power injection for base load condition.

In order to verify the self-sensitivity $d|V| / d Q$ of node $1175,1.0$ p.u of its own reactive power load with step 0.1 p.u are injected into the bus 1175 . The voltage magnitudes of node 1175 are then predicted by multiplying the selfsensitivity $d|V| / d Q$ at the base load condition with the amount of the reactive power injection. These voltages are then compared with the actual voltages obtained via the results of power flow with the reactive power injection. A similar verification is repeated for the largest cross-sensitivity $d|V| / d Q$ of node 1175.1 .0 p.u of node 1165 reactive power load with step 0.1 p.u are injected into the bus 1165. Fig.7 (a) and Fig.7 (b) show the verification of $d|V| / d Q$ sensitivity of bus 1175 due to reactive power injected at bus 1175 and bus 1165 respectively

The errors in the voltage at bus 1175 are summarized in Table I. It can be seen as the power injection increases, the errors in the results increase. Those errors are common in the sensitivities that are used for nonlinear power systems. However, the errors at a large amount change are still very small which demonstrates good accuracy. It can be also seen that the errors in the self-sensitivities are smaller than the errors in the cross-sensitivity.

The errors in the voltage at bus 1175 due to power injections at bus 1115 are summarized in Table II. The results show the accuracy of the proposed method in estimating the voltage of a bus far from the bus with power injections. Those errors are also still very small and common in the sensitivities that are used for nonlinear power systems. This accuracy of the proposed method is also coinciding with the high computation speed shown in the next subsections.

\section{A Simple Application of the proposed sensitivity method}

In distribution power network operation, it is required to maintain the system voltages within normal limits. Once the voltage of any node is below its lower limit Vmin, appropriate actions should be taken. In this subsection, a simple voltage control is presented to demonstrate the applicability of the proposed sensitivity method.

In this scenario, feeder 4 of the study system is operated at high loading condition such that some voltages are violated. It was found that bus 1175 has a lower voltage magnitude. By assuming that network operators attempt to correct the voltages starting from the most violated voltage and by injection reactive power at bus 1175 , the reactive power compensation amount can be determined by:

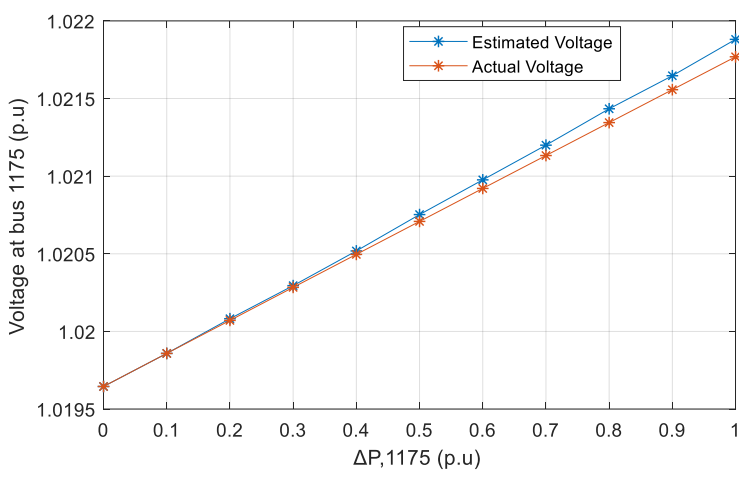

(a)

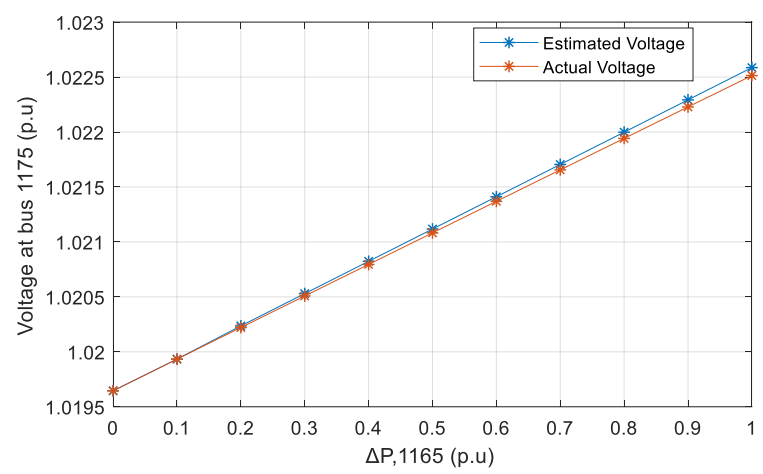

(b)

Fig.6. Verification of $d|V| / d P$ sensitivity of bus 1175 due to active power injected at (a) bus 1175 (b) bus 1165

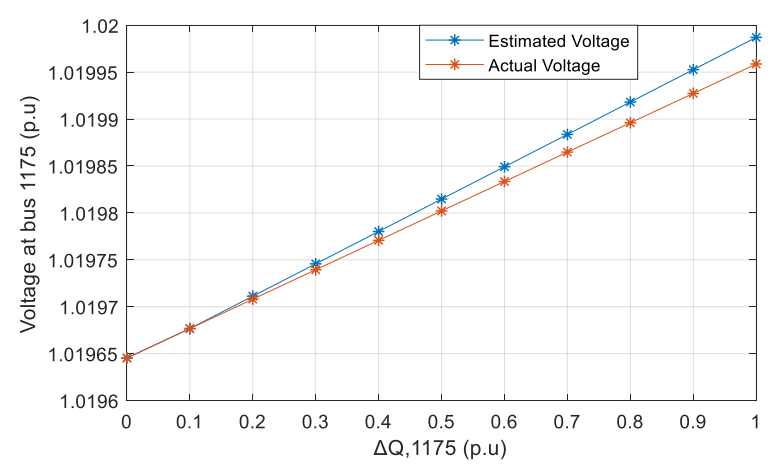

(a)

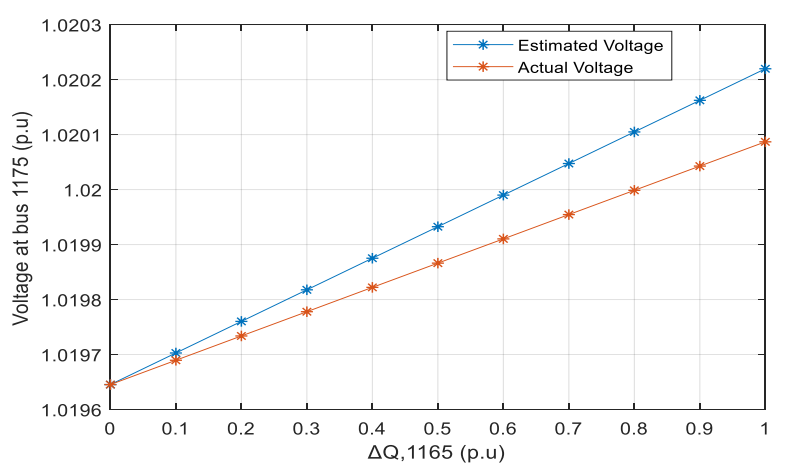

(b)

Fig.7. Verification of $d|V| / d Q$ sensitivity of bus 1175 due to reactive power injected at bus (a) bus 1175 (b) bus 1165

$$
\begin{aligned}
& \Delta Q_{1175}=\frac{\Delta V_{1175}}{d|V|_{1175} / d Q_{1175}} \\
= & \frac{\mathrm{V}-\mathrm{V}_{o}}{d|V|_{1175} / d Q_{1175}}
\end{aligned}
$$


TABLE.1: The ERrors In The Voltage At Bus 1175

\begin{tabular}{|l|l|l|l|l|}
\hline \multirow{2}{*}{$\begin{array}{l}\Delta \mathrm{Q} \text { O Or } \\
\text { (p.u) }\end{array}$} & \multicolumn{4}{|c|}{ error $(\%)$ in the voltage 1175} \\
\cline { 2 - 5 } & Self-sensitivity to: & \multicolumn{1}{c|}{ Cross-sensitivity to: } \\
\cline { 2 - 5 } & $P_{1175}$ & $Q_{1175}$ & $P_{1165}$ & $Q_{1165}$ \\
\hline 0.1 & 0 & 0 & 0 & 0 \\
\hline 0.2 & $6.68 \times 10^{-08}$ & $1.73 \times 10^{-09}$ & $2.33 \times 10^{-05}$ & $1.29 \times 10^{-05}$ \\
\hline 0.3 & $1.06 \times 10^{-05}$ & $3.08 \times 10^{-06}$ & $1.47 \times 10^{-03}$ & $2.59 \times 10^{-05}$ \\
\hline 0.4 & $1.09 \times 10^{-05}$ & $6.16 \times 10^{-06}$ & $2.10 \times 10^{-03}$ & $3.90 \times 10^{-05}$ \\
\hline 0.5 & $2.18 \times 10^{-05}$ & $9.25 \times 10^{-06}$ & $2.75 \times 10^{-03}$ & $5.20 \times 10^{-05}$ \\
\hline 0.6 & $4.31 \times 10^{-05}$ & $1.23 \times 10^{-05}$ & $3.42 \times 10^{-03}$ & $6.50 \times 10^{-05}$ \\
\hline 0.7 & $5.42 \times 10^{-05}$ & $1.54 \times 10^{-05}$ & $4.11 \times 10^{-03}$ & $7.80 \times 10^{-05}$ \\
\hline 0.8 & $6.53 \times 10^{-05}$ & $1.85 \times 10^{-05}$ & $4.82 \times 10^{-03}$ & $9.10 \times 10^{-05}$ \\
\hline 0.9 & $8.70 \times 10^{-05}$ & $2.16 \times 10^{-05}$ & $5.56 \times 10^{-03}$ & $1.04 \times 10^{-04}$ \\
\hline 1.0 & $8.79 \times 10^{-05}$ & $2.47 \times 10^{-05}$ & $6.31 \times 10^{-03}$ & $1.17 \times 10^{-04}$ \\
\hline
\end{tabular}

TABle.2: The ERrors In The Voltage At Bus 1175 Due To Power INJECTIONS AT Bus 1115

\begin{tabular}{|l|l|l|l|l|l|}
\hline \multirow{2}{*}{$\begin{array}{l}\text { Sens. } \\
\text { to: }\end{array}$} & \multicolumn{5}{|c|}{ error $(\%)$ in the voltage 1175} \\
\cline { 2 - 6 } & 0.1 & 0.2 & 0.3 & 0.4 & 0.5 \\
\cline { 2 - 6 } & $1.8 \times 10^{-09}$ & $5.5 \times 10^{-05}$ & $2.4 \times 10^{-03}$ & $3.1 \times 10^{-03}$ & $4.0 \times 10^{-03}$ \\
\hline$P_{1115}$ & $1.5 \times 10^{-09}$ & $4.3 \times 10^{-05}$ & $5.7 \times 10^{-05}$ & $8.2 \times 10^{-05}$ & $2.7 \times 10^{-04}$ \\
\hline$Q_{1115}$ & $1.5 \mathrm{P}(\mathrm{p} . \mathrm{u})$ \\
\hline
\end{tabular}

Where $\Delta V_{1175}$ is the change amount of the voltage magnitude that should be compensated to correct the voltage at bus $1175 . \mathrm{V}_{o}$ is considered as the minimum voltage limit (equals to 0.98 p.u in this work). $V$ is considered as the node voltage before implementing control actions.

From the results, it was found that voltage of node 1175 before performing control action was 0.9785 p.u and the sensitivity $d|V|_{1175} / d Q_{1175}=0.7386$ p.u. Using (14), it was found that the reactive power compensation amount $\Delta Q_{1175}$ should be 0.002 p.u. Fig. 8 shows some of lowest voltages of feeder 4 before and after performing the control action. It is clear that the proposed sensitivity approach successfully works for voltage control.

\section{Calculation speed}

In terms of the calculation time, the proposed method is analyzed for the case shown in subsection A of simulation results. It was found that the sensitivity analysis time is around $0.48 \mathrm{~s}$. This time is compared with the calculation speed obtained from a typical method via the inverse of the updated Jacobian matrix obtained from the load flow solution. It takes about $0.65 \mathrm{~s}$ to obtain the sensitivities of the same case. This is due to the iteration process that is required for the later approach. Accordingly, the proposed method was able to reduce the computation time by $26.2 \%$. These times were obtained using an i7-8850 H CPU @ 2.60 GHz laptop.

It is worth mentioning that the reduction in the computing time will be higher in case of large practical systems. Moreover in the context of the optimization- based voltage control techniques, the saving computing time will be much higher.

\section{CONCLUSIONS}

In this work, a fast approach for computing the voltage sensitivity coefficients with respect to nodal power injections are presented. The method uses the direct analytical derivation to obtain the sensitivities. The formulas show that the approach is based on network admittance matrix and does not require extensive computing time, which is an advantage to be implemented in online applications.

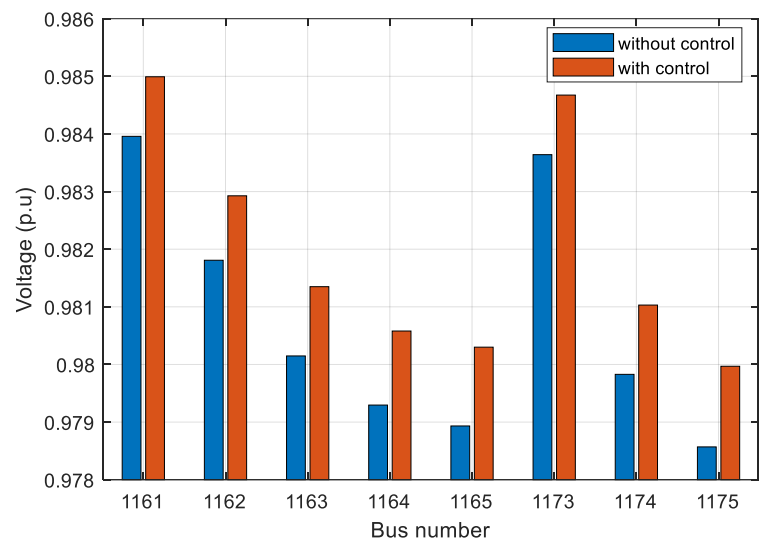

Fig.8. Voltages of some buses of feeder 4 of the test system with and without control

The proposed method has been validated on a $11 \mathrm{kv}, 75$-bus distribution grid. The numerical values of the sensitivity matrices validated the computation of the coefficients. Verification of the self-sensitivity and the largest cross sensitivity of the critical bus, bus 1175 , are also investigated in details. It is shown that the errors of the proposed sensitivities are very small which demonstrates good accuracy. The simple voltage control scenario presented in this paper demonstrated the potential application of the proposed sensitivity method.

A further extension would be to include different types of control variables (continuous and discrete) in the sensitivity analysis.

\section{ACKNOWLEDGMENT}

This work was supported by the National Priorities Research Program under Grant 11S-1125-170027 from the Qatar National Research Fund (a member of Qatar Foundation).

\section{REFERENCES}

[1] M. Begovic and A. Phadke, "Control of voltage stability using sensitivity analysis," Power Systems, IEEE Transactions on, vol. 7, no. 1, pp. $114-123,1992$

[2] H. Mehrjerdi., S. Lefebvre., M.Saad, and D. Asber. « Coordinated Control Strategy Considering Effect of Neighbourhood Compensation for Voltage Improvement in Transmission Systems ». IEEE Trans. Power Syst., vol. 28, no.4, pp. 4507-4515, Nov 2013.

[3] Q. Zhou and J. Bialek, "Simplified calculation of voltage and loss sensitivity factors in distribution networks," in Proc. of the 16th Power Systems Computation Conference, Glasgow, Scotland, 2008.

[4] S. Conti, S. Raiti, and G. Vagliasindi, "Voltage sensitivity analysis in radial mv distribution networks using constant current models," in Industrial Electronics (ISIE), 2010 IEEE International Symposium on. IEEE, pp. 2548-2554, 2010

[5] D. Khatod, V. Pant, and J. Sharma, "A novel approach for sensitivity calculations in the radial distribution system," Power Delivery, IEEE Transactions on, vol. 21, no. 4, pp. 2048-2057, 2006.

[6] R. Gurram and B. Subramanyam, "Sensitivity analysis of radial distribution network-adjoint network method," International Journal of Electrical Power \& Energy Systems, vol. 21, no. 5, pp. 323-326, 1999.

[7] G. Valverde and T. Van Cutsem, "Model predictive control of voltages in active distribution networks. "IEEE Transactions on Smart Grid, vol.4, no. 4, pp. 2152-2161, 2013.

[8] United Kingdom Generic Distribution Network (UKGDS). [Online]. Available: http://sedg.ac.uk 